\title{
Relaxation of muon spins in molecular nanomagnets
}

\author{
T. Lancaster, ${ }^{1, *}$ S. J. Blundell, ${ }^{1}$ F. L. Pratt,${ }^{2}$ I. Franke, ${ }^{1}$ A. J. Steele, ${ }^{1}$ P. J. Baker, ${ }^{2}$ Z. Salman, ${ }^{3}$ C. Baines,${ }^{3}$ I. Watanabe, ${ }^{4}$ \\ S. Carretta, ${ }^{5}$ G. A. Timco, ${ }^{6}$ and R. E. P. Winpenny ${ }^{6}$ \\ ${ }^{1}$ Clarendon Laboratory, Department of Physics, Oxford University, Parks Road, Oxford OX1 3PU, United Kingdom \\ ${ }^{2}$ ISIS Facility, Rutherford Appleton Laboratory, Chilton, Oxfordshire OX11 OQX, United Kingdom \\ ${ }^{3}$ Laboratory for Muon Spin Spectroscopy, Paul Scherrer Institut, CH-5232 Villigen PSI, Switzerland \\ ${ }^{4}$ Muon Science Laboratory, RIKEN, 2-1 Hirosawa, Wako, Saitama 351-0198, Japan \\ ${ }^{5}$ Dipartmento di Fisica, Università di Parma, I-43100 Parma, Italy \\ ${ }^{6}$ Department of Chemistry, University of Manchester, Oxford Road, Manchester M13 9PL, United Kingdom
}

(Received 16 March 2010; published 28 April 2010)

\begin{abstract}
We address the cause of the unusual muon spin-relaxation $\left(\mu^{+} \mathrm{SR}\right)$ results on molecular nanomagnets (MNMs). Through measurements on protonated and deuterated samples of the $\mathrm{MNMs}_{7} \mathrm{Cr}_{7} \mathrm{Mn}(S=1)$ and $\mathrm{Cr}_{8}$ $(S=0)$, we show that the muon spin for $S \neq 0$ MNMs is relaxed via dynamic fluctuations of the electronic spins. A freezing out of dynamic processes occurs on cooling and at low temperatures the muon spins are relaxed by the electronic spins which themselves are dephased by incoherent nuclear-field fluctuations. We observe a transition to a state of static magnetic order of the MNM electronic spins in $\mathrm{Cr}_{7} \mathrm{Mn}$ below $2 \mathrm{~K}$.
\end{abstract}

DOI: 10.1103/PhysRevB.81.140409

PACS number(s): 75.50.Xx, 76.75.+i

Molecular nanomagnets (MNMs) (Ref. 1) comprise clusters of exchange-coupled transition-metal ions which often have a negative anisotropy constant favoring a ground state with a large eigenvalue of the electronic spin component $S_{z}$. These systems have been widely studied in recent years, most recently in anticipation of their deployment as elements of quantum computers ${ }^{2,3}$ although much interest also centers on the quantum tunnelling of the magnetization (QTM) which takes place when the magnetic energy levels are at resonance. ${ }^{1}$ The theory of QTM is based on the LandauZener paradigm ${ }^{4}$ of energy levels brought into resonance by a time-dependent field. The precise details of the mechanism for QTM in MNMs remain unresolved and while pairwise dipole interactions between spins are certainly important ${ }^{5}$ a promising suggestion is that resonance is achieved as a result of the stochastically varying magnetic field $\boldsymbol{B}_{\mathrm{n}}(t)$, which arises from the incoherent fluctuations of nuclear moments surrounding the transition-metal ions ${ }^{6}$ (a typical MNM contains $\sim 10^{2}$ protons due to the organic ligands).

MNMs have been successfully probed using techniques such as magnetization, ${ }^{7}$ heat capacity, ${ }^{8}$ neutron scattering,,${ }^{9,10}$ and electron spin resonance (ESR). ${ }^{3}$ In contrast, measurements made using muon spin relaxation $\left(\mu^{+} \mathrm{SR}\right)$ have proven difficult to interpret. Although initially it was thought that QTM should be measurable by implanting muons into MNMs, ${ }^{11-13}$ the unambiguous detection of this effect proved elusive. ${ }^{14}$ Instead, $\mu^{+}$SR spectra obtained on high spin systems appeared to arise from dynamic fluctuations of a local magnetic field distribution at the muon sites, which persisted down to dilution refrigerator temperatures. ${ }^{12-14}$ Muon results on MNM systems all showed similar behavior ${ }^{14}$ but it was unclear whether the muon was probing the intrinsic behavior of the large electronic spin or some residual effect. It was argued recently by Keren et al. ${ }^{15}$ that $\mu^{+} \mathrm{SR}$ is sensitive to the relaxation of the MNM electronic spins caused by the incoherent fluctuations of nuclear moments surrounding the metal ions. If this is the case then it makes the muon a valuable probe of the potential mechanism behind QTM. In order to address the question of what the muon probes in
MNM systems we have made identical $\mu^{+} \mathrm{SR}$ measurements on protonated and deuterated samples of $\mathrm{Cr}_{7} \mathrm{Mn}(S=1)$ and $\mathrm{Cr}_{8}(S=0)$ (Refs. 16-19) (structure shown in Fig. 1). We show (i) that the muon is controlled by the large electronic spin in an MNM; (ii) deuteration leads to a significant increase in the $\mu^{+} S R$ relaxation rate at low temperature in $\mathrm{Cr}_{7} \mathrm{Mn}$, implying that muons probe the relaxation of large electronic spins by the random magnetic fields due to the nuclei, and (iii) that upon cooling, a magnetic ground state is reached by a freezing out of dynamic processes that leads to magnetic order in $\mathrm{Cr}_{7} \mathrm{Mn}$ below $2 \mathrm{~K}$.

In a $\mu^{+} \mathrm{SR}$ experiment, ${ }^{20}$ spin-polarized positive muons are stopped in a target sample. The time evolution of the muon spin polarization is probed via the positron decay asymmetry function $A(t)$ to which it is proportional. Our $\mu^{+}$SR measurements were made on the ARGUS instrument at the RIKEN-RAL facility, ISIS, Rutherford Appleton Laboratory, U.K. and on the low temperature facility (LTF) and general purpose spectrometer (GPS) instruments at the Swiss Muon Source $(\mathrm{S} \mu \mathrm{S})$, Paul Scherrer Institut, $\mathrm{CH}$. Powder samples of the materials were packed in $\mathrm{Ag}$ foil and mounted on a $\mathrm{Ag}$ backing plate in ${ }^{3} \mathrm{He}$ and ${ }^{4} \mathrm{He}$ cryostats. Typical spectra measured for $\mathrm{Cr}_{7} \mathrm{Mn}$ and $\mathrm{Cr}_{8}$ are shown in Fig. 1 . Above $T \approx 2 \mathrm{~K}$ the spectra for all materials differ depending on whether protonated or deuterated (Fig. 1).

To compare the $\mathrm{Cr}_{7} \mathrm{Mn}$ and $\mathrm{Cr}_{8}$ systems, measurements were made over the temperature range $2 \leq T \leq 100 \mathrm{~K}$. In this regime the spectra for $S=1 \mathrm{Cr}_{7} \mathrm{Mn}$ [Fig. 1(a)] were found to be described by the relaxation function $A(t)=A_{1} \exp (-\sqrt{\lambda t})$ $+A_{\mathrm{bg}}$, where $A_{\mathrm{bg}}$ accounts for any background contribution from muons that stop in the sample holder or cryostat tails. This behavior is typical of that observed previously in MNM materials ${ }^{12,13,15}$ and arises because of the complex dynamic distribution of local fields within the material sampled by the muon ensemble. The monotonic relaxation and the fact that the muons could not be decoupled with an applied magnetic field up to $0.6 \mathrm{~T}$ places the relaxation in the fast-fluctuation limit. $^{21}$ 


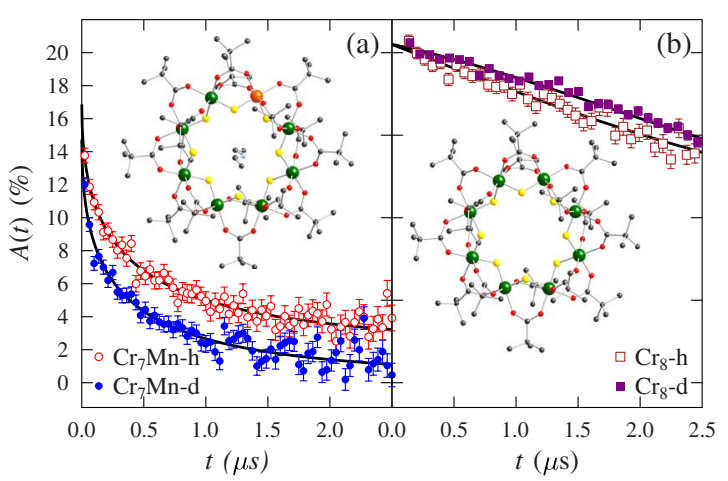

FIG. 1. (Color online) $\mu^{+} \mathrm{SR}$ spectra for protonated and deuterated (a) $\mathrm{Cr}_{7} \mathrm{Mn}$ and (b) $\mathrm{Cr}_{8}$ materials, measured at $T=4.5 \mathrm{~K}$. Inset: structures of the molecules.

The spectra measured for the $S=0 \mathrm{Cr}_{8}$ samples are quite different [Fig. 1(b)]. In this case the relaxation rate is far smaller and resembles a Kubo-Toyabe (KT) function $f_{\mathrm{KT}}(\Delta t)$ with $\Delta=\gamma_{\mu} \sqrt{\left\langle B^{2}\right\rangle}$ where $\gamma_{\mu}=2 \pi \times 135.5 \mathrm{MHz} \mathrm{T}^{-1}$ is the muon gyromagnetic ratio and $B$ is the local magnetic field at a muon site. ${ }^{21}$ However, the KT function, which describes relaxation due to static random magnetic fields at the muon sites, could not adequately describe all of the data. This is probably to be expected in a complex material such as a MNM where many inequivalent classes of muon sites occur and lead to a distribution of second moments $p(\Delta)$. The resulting muon relaxation is obtained by averaging the KT function over this distribution. For simplicity we modeled the relaxation by taking $p(\Delta)$ to be a uniform distribution up to a maximum $\Delta_{0}$. This one-parameter model successfully fitted the data for both materials over the entire time range. The fitted values of $\Delta_{0}$ are shown in Fig. 2(a) and are quite $T$ independent and take average values $\Delta_{0}=0.56(1) \mathrm{MHz}$ for $\mathrm{Cr}_{8}-h$ and 0.48 (1) MHz for $\mathrm{Cr}_{8}-d$. Our conclusion from these measurements is that the muon is sensitive to the disordered nuclear moments in $\mathrm{Cr}_{8}$. This is confirmed by the application of a small longitudinal magnetic field which quenches the relaxation. The larger $\Delta_{0}$ in $\mathrm{Cr}_{8}-h$ compared to $\mathrm{Cr}_{8}-d$ reflects (albeit partially) the larger moment of the proton. Most importantly, the dramatic difference between the measured spectra and relaxation rates for $S=0 \mathrm{Cr}_{8}$ and $S=1 \quad \mathrm{Cr}_{7} \mathrm{Mn}$ samples (Fig. 1) strongly suggests that the muon response in MNM systems with $S \neq 0$ stems from dynamic fluctuations of the electronic spin. In the absence of an electron spin in $\mathrm{Cr}_{8}$, the muon spin is relaxed by quasistatic disordered nuclear moments.

We now turn to the role of the nuclei in the spin relaxation process in $S=1$ MNMs. The temperature dependence of the relaxation rate $\lambda$ for the protonated $\left(\lambda^{h}\right)$ and deuterated $\left(\lambda^{d}\right)$ $\mathrm{Cr}_{7} \mathrm{Mn}$ samples is shown in Fig. 2(a). These measurements were made with a magnetic field of $1 \mathrm{mT}$ applied parallel to the initial muon spin direction which was intended to quench any residual relaxation of the muon spin caused directly by fluctuating nuclear spins as seen in the $\mathrm{Cr}_{8}$ samples. On cooling below $T \sim 50 \mathrm{~K}$, the relaxation rate $\lambda$ increases before saturating below $\sim 10 \mathrm{~K}$. with the onset of the increase and the saturation occurring at similar values of $T$ for both materials. This $T$ dependence is common to nearly all MNM

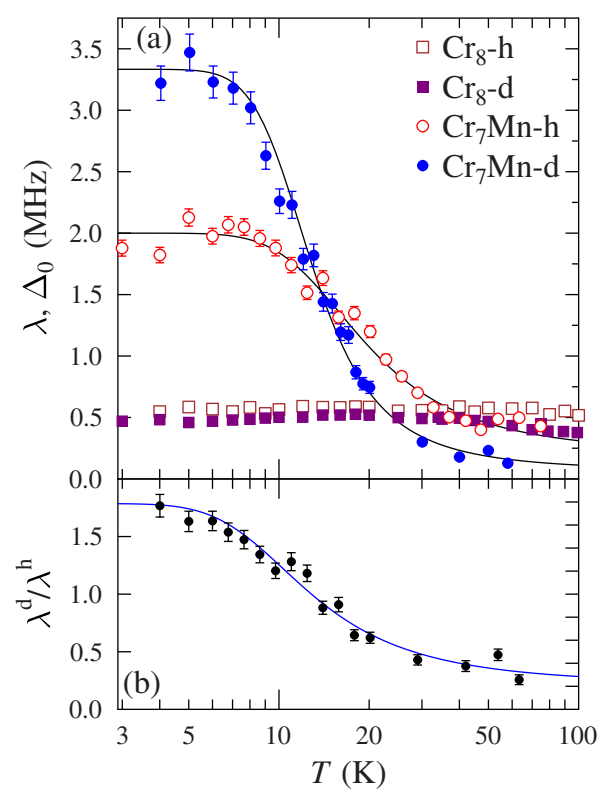

FIG. 2. (Color online) (a) Temperature evolution of the relaxation rates $\lambda$ for $\mathrm{Cr}_{7} \mathrm{Mn}$ and $\Delta_{0}$ for $\mathrm{Cr}_{8}$. The lines are fits to a phenomenological equation (see main text). (b) Ratio of the $\mathrm{Cr}_{7} \mathrm{Mn}-h$ and $-d$ relaxation rates. The line is a guide to the eye.

systems that have been previously measured with $\mu^{+}$SR (Refs. 12-15) and is discussed in more detail below. At high $T$ we see that $\lambda^{d}>\lambda^{h}$. It is likely that at these high temperatures the electronic spins are fluctuating very fast and are at least partially motionally narrowed from the spectra. Upon cooling the increase in $\lambda$ is greater for the deuterated sample, with $\lambda^{d}$ becoming greater than $\lambda^{h}$ below $\approx 15 \mathrm{~K}$. Most significantly the saturation of the relaxation at $T \lesssim 10 \mathrm{~K}$ occurs with $\lambda^{d}>\lambda^{h}$. The $T$ dependence of the ratio $\lambda^{d} / \lambda^{h}$ [Fig. 2(b)] which increases on cooling tends to $\approx 1.7$ at the lowest temperature.

The low-temperature results may be understood using the model of $\mu^{+}$SR in MNM systems proposed by Keren et al. ${ }^{15}$ Here a muon spin $\boldsymbol{I}$ is coupled to a single MNM electronic spin $S$ and dynamic fluctuations of the local protons cause $S$ to experience a nuclear stochastic field $\boldsymbol{B}_{\mathrm{n}}(t)$, along with any externally applied field $\boldsymbol{B}_{\mathrm{a}}$. This system may be described by the resulting Hamiltonian $H=g_{\mathrm{s}} \mu_{\mathrm{B}} \boldsymbol{S} \cdot\left[\boldsymbol{B}_{\mathrm{a}}+\boldsymbol{B}_{\mathrm{n}}(t)\right]+\hbar \gamma_{\mu} \mathbf{I} \cdot\left[\boldsymbol{B}_{\mathrm{a}}\right.$ $+\mathbf{F S}]$, where $\mathbf{F}$ couples the muon and electronic spin. We ignore the direct response of the muon to the stochastic field, which we know to be small from the $\mathrm{Cr}_{8}$ results. The spin relaxation resulting from this model may be simply understood. The magnetic field distribution experienced by a particular spin population is characterized by a correlation function whose Fourier transform (known as the spectral weight) will be proportional to the spin-relaxation rate. We assume that the stochastic magnetic field $\boldsymbol{B}_{\mathrm{n}}(t)$ experienced by the MNM spin due to the nuclei will be described by $\left\langle\boldsymbol{B}_{\mathrm{n}}(t) \boldsymbol{B}_{\mathrm{n}}(0)\right\rangle=\left\langle B_{n}^{2}\right\rangle \exp \left(-t / \tau_{\mathrm{n}}\right)$, where $\tau_{\mathrm{n}}$ is the correlation time of the nuclear stochastic field. A Fourier transform reveals that the nuclear fields will relax the electron spins at a rate $^{15} 1 / \tau_{\mathrm{e}} \propto\left\langle B_{\mathrm{n}}^{2}\right\rangle \tau_{\mathrm{n}}$. To find the relaxation rate of the muon spin due to the fluctuations of the electronic spins, we again assume that the correlation function takes the form 
$\left\langle\boldsymbol{B}_{\mu}(t) \boldsymbol{B}_{\mu}(0)\right\rangle=\left\langle B_{\mu}^{2}\right\rangle \exp \left(-t / \tau_{\mathrm{e}}\right)$, where $\boldsymbol{B}_{\mu}$ is the effective local field at the muon site due to coupling to the large spin $S$ and $\tau_{\mathrm{e}}$ is the correlation time for the electronic spins. Taking a further Fourier transform gives the $\mu^{+} \mathrm{SR}$ relaxation rate $^{15,21}$ as $\lambda=\gamma_{\mu}^{2}\left\langle B_{\mu}^{2}\right\rangle \tau_{\mathrm{e}} /\left[1+\left(\gamma_{\mu}\left|B_{\mathrm{a}}\right| \tau_{\mathrm{e}}\right)^{2}\right]$ which in the limit of small applied magnetic fields becomes

$$
\lambda=\gamma_{\mu}^{2}\left\langle B_{\mu}^{2}\right\rangle \tau_{\mathrm{e}} \propto \frac{\left\langle B_{\mu}^{2}\right\rangle}{\left\langle B_{\mathrm{n}}^{2}\right\rangle} \frac{1}{\tau_{\mathrm{n}}} .
$$

The primary effect of swapping protons for deuterons might be expected to be the factor 3.26 decrease in the size of the nuclear moments experienced by the electronic spin, reducing the second moment of the field distribution due to the electronic spin $\left\langle B_{\mu}^{2}\right\rangle$. There is good evidence for this reduction from ESR (Ref. 3) in the similar system $\mathrm{Cr}_{7} \mathrm{Ni}$, where it was found that $1 / T_{2}$ decreased upon deuteration, demonstrating that the electronic spins are directly relaxed by proton fluctuations. Equation (1) shows that a decrease in $\left\langle B_{\mathrm{n}}^{2}\right\rangle$ will lead to an increase in the muon relaxation rate $\lambda$. The larger magnitude of $\lambda$ for the $\mathrm{Cr}_{7} \mathrm{Mn}-d$ sample at low temperatures is therefore consistent with the electronic spin being dephased by the nuclei. Since the electronic spins, and hence $\left\langle B_{\mu}^{2}\right\rangle$, have the same magnitude in both materials, then we have $\lambda^{d} / \lambda^{h}=\left\langle B_{\mathrm{n}=h}^{2}\right\rangle \tau_{\mathrm{n}=h} /\left\langle B_{\mathrm{n}=d}^{2}\right\rangle \tau_{\mathrm{n}=d}$. For our systems, we might expect that $\left\langle B_{\mathrm{n}}^{2}\right\rangle \propto \gamma_{\mathrm{n}}^{2} I_{\mathrm{n}}\left(I_{\mathrm{n}}+1\right)$ (Ref. 22) and $\tau_{n}$ $\propto 1 /\left[\gamma_{\mathrm{n}} \sqrt{I_{\mathrm{n}}\left(I_{\mathrm{n}}+1\right)}\right]$ [where the latter prediction results from considering the Overhauser field in an MNM (Ref. 23)], leading to the prediction that $\lambda^{d} / \lambda^{h}=3.99$. The measured increase at low temperatures is, however, a factor of $\approx 1.7$. A discrepancy is not surprising, particularly given that the ratio of measured $\Delta_{0}$ factors for $\mathrm{Cr}_{8}-h$ and $-d$ samples is less than the factor predicted above for the second moments. However, this alone is not enough to explain the size of the discrepancy and this may provide evidence that the reorientations of the electronic moments caused by the nuclei are not isotropic.

Finally we address the characteristic temperature dependence of $\lambda$ observed in all $S \neq 0$ MNM systems. It is generally found in MNMs (Ref. 14) that on decreasing the temperature from $T \sim 100 \mathrm{~K}$ there is an increase in $\lambda$ with the relaxation rate leveling off to some value $\lambda_{\text {sat }}$ below $\sim 10 \mathrm{~K}$. This behavior may be explained by involving two competing dynamic relaxation processes, one dominant at high temperatures and described by a strongly temperature-dependent correlation time $\tau_{s}(T)$ and one, dominant at low temperatures described by a weakly $T$-dependent correlation time $\tau_{\mathrm{w}}$. In the presence of two competing processes, that with the shorter correlation time dominates, giving the smaller relaxation rate (since $\lambda \propto 1 / \tau)$. At high temperature, therefore, we have $\tau_{\mathrm{s}}(T) \ll \tau_{\mathrm{w}}$ which results in a strongly $T$-dependent relaxation which we can crudely model phenomenologically with $\lambda=C \exp (U / T)$, where $U$ represents an energy barrier. At low temperatures when some of the $T$-dependent relaxation channels have been frozen out, $\tau_{\mathrm{w}} \ll \tau_{\mathrm{s}}(T)$ and we have $\lambda \sim \lambda_{\text {sat }}$. This behavior results in a phenomenological fitting function $1 / \lambda(T)=1 / \lambda_{\text {sat }}+1 /[C \exp (U / T)]$ which has been used previously to characterize these MNM systems. ${ }^{14}$ Fitting this formula to our data [Fig. 2(a)] yields

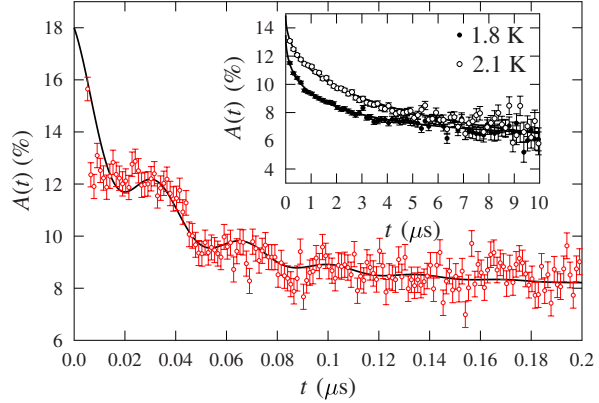

FIG. 3. (Color online) Example spectrum measured at $0.5 \mathrm{~K}$ for $\mathrm{Cr}_{7} \mathrm{Mn}-h$ (at $\mathrm{S} \mu \mathrm{S}$ ) showing heavily damped oscillations with a frequency of $\gamma_{\mu} B /(2 \pi) \sim 30 \mathrm{MHz}$. Inset: spectra from ISIS show a discontinuous change upon cooling below $2 \mathrm{~K}$.

$C^{h}=0.23(3) \mathrm{MHz}, \quad U^{h}=46(4) \mathrm{K}, \quad \lambda_{\mathrm{sat}}^{h}=2.00(4) \mathrm{MHz}, C^{d}$ $=0.070(1) \mathrm{MHz}, U^{d}=51(3) \mathrm{K}$, and $\lambda_{\mathrm{sat}}^{\mathrm{d}}=3.3(1) \mathrm{MHz}$.

There is an intriguing similarity between the muon results in MNM systems and those in some inorganic materials, such as $\mathrm{Ca}_{3} \mathrm{Co}_{2-x} \mathrm{Mn}_{x} \mathrm{O}_{6},{ }^{24}$ where the physics involves significant single-ion anisotropy and a complex (often glassy) freezing out of dynamic processes. It is probable that the relaxation of the electronic spins we probe in MNMs is explainable within the same framework. At high temperatures magnetoelastic interactions provide the main relaxation mechanism for the electronic degrees of freedom. Our data show $U^{h} \approx U^{d}$ since, if the $T$ dependence is due to spinphonon coupling through the modulation of local crystal fields, this barrier height should only depend on the electronic energy level structure. As the temperature is decreased, some relaxation channels will be frozen out, increasing the correlation time of the electronic moments. ${ }^{25}$ The low-temperature channel that gives rise to the temperatureindependent relaxation $\lambda_{\text {sat }}$ seen in $\mu^{+}$SR would appear to be the relaxation of the electronic spins by the nuclear fluctuations, allowing us to identify $\tau_{\mathrm{w}}$ with $\tau_{\mathrm{e}}$, the electronic correlation time discussed above. This situation is similar to the electronic $T_{2}$ which is dominated by phononic contributions at high temperatures and by nuclear contributions at low temperature. $^{26}$ This is not the case for the electronic $1 / T_{1}$ which is phonon dominated down to $\sim 2 \mathrm{~K}$. This difference between $1 / T_{1}$ and $1 / T_{2}$ for ESR suggests that the nuclei contribute to the secular part of the relaxation, that is, the relaxation of the electron spins due to a spread in the net magnetic field at the spin sites. ${ }^{3,22}$

In order to further probe the freezing out of the dynamics in $\mathrm{Cr}_{7} \mathrm{Mn}$, measurements were made down to $20 \mathrm{mK}$ using the LTF instrument at $\mathrm{S} \mu \mathrm{S}$. At the lowest temperatures heavily damped oscillations are observed at early times (Fig. 3 ) in both $\mathrm{Cr}_{7} \mathrm{Mn}-h$ and $-d$. Oscillations are usually caused in muon spectra by quasistatic magnetic order, causing a coherent precession of muon spins. The observed oscillations show little temperature dependence in the range $0.02 \leq T$ $\leq 1 \mathrm{~K}$ and are identical for $-h$ and $-d$ samples. For measurements made in the same temperature range at ISIS, oscillations are not discernible due to the resolution limit set by the ISIS muon pulse width. However, the spectra do show a discontinuous change around $T=2 \mathrm{~K}$ (inset Fig. 3) with a 
loss of initial asymmetry and a sharp increase in the apparent relaxation rate observed below this temperature. There is also significant magnetic hysteresis on the application and removal of applied magnetic fields below $2 \mathrm{~K}$. From these measurements we estimate that a transition to a state of static magnetic order takes place at $T=1.9(1) \mathrm{K}$. The heavily damped nature of the oscillations and the $\mathrm{Cr}_{8}$ results suggest that there are many magnetically inequivalent muon sites in the system. The results demonstrate that the intermolecular exchange $J$ is nonzero in the $S=1$ system and, using mean- field theory ${ }^{27}$ (assuming $z=6$ nearest neighbors) we estimate $J / k_{\mathrm{B}}=3 T_{\mathrm{C}} / 2 z S(S+1) \approx 0.2 \mathrm{~K}$.

This work was carried out at $\mathrm{S} \mu \mathrm{S}$ and the RIKEN-RAL facility (ISIS) supported by a beam time allocation from the Science and Technology Facilities Council. We are grateful to Alex Amato for experimental assistance, Chris Wedge for useful discussion and the EPSRC (U.K.) for financial support. This work is partially supported by the European FP7ICT FET Open “MolSpinQIP” project, Contract No. 211284. *t.lancaster1@physics.ox.ac.uk

${ }^{1}$ D. Gatteschi, R. Sessoli, and J. Villain, Molecular Nanomagnets (Oxford University Press, New York, 2006).

${ }^{2}$ M. N. Leuenberger and D. Loss, Nature (London) 410, 789 (2001).

${ }^{3}$ A. Ardavan, O. Rival, J. J. L. Morton, S. J. Blundell, A. M. Tyryshkin, G. A. Timco, and R. E. P. Winpenny, Phys. Rev. Lett. 98, 057201 (2007).

${ }^{4}$ L. D. Landau, Phys. Z. Sowjetunion 2, 46 (1932); C. Zener, Proc. R. Soc. London, Ser. A 137, 696 (1932).

${ }^{5}$ A. Morello, P. C. E. Stamp, and I. S. Tupitsyn, Phys. Rev. Lett. 97, 207206 (2006).

${ }^{6}$ N. V. Prokof'ev and P. C. E. Stamp, Phys. Rev. Lett. 80, 5794 (1998).

${ }^{7}$ A. Cornia, D. Gatteschi, and R. Sessoli, Coord. Chem. Rev. 219221, 573 (2001).

${ }^{8}$ F. Fominaya, J. Villain, P. Gandit, J. Chaussy, and A. Caneschi, Phys. Rev. Lett. 79, 1126 (1997).

${ }^{9}$ M. Hennion, L. Pardi, I. Mirebeau, E. Suard, R. Sessoli, and A. Caneschi, Phys. Rev. B 56, 8819 (1997).

${ }^{10}$ R. Caciuffo, T. Guidi, G. Amoretti, S. Carretta, E. Liviotti, P. Santini, C. Mondelli, G. Timco, C. A. Muryn, and R. E. P. Winpenny, Phys. Rev. B 71, 174407 (2005).

${ }^{11}$ A. Lascialfari, Z. H. Jang, F. Borsa, P. Carretta, and D. Gatteschi, Phys. Rev. Lett. 81, 3773 (1998).

${ }^{12}$ Z. Salman, A. Keren, P. Mendels, V. Marvaud, A. Scuiller, M. Verdaguer, J. S. Lord, and C. Baines, Phys. Rev. B 65, 132403 (2002).
${ }^{13}$ S. J. Blundell et al., Synth. Met. 133-134, 531 (2003).

${ }^{14}$ T. Lancaster et al., J. Phys.: Condens. Matter 16, S4563 (2004).

${ }^{15}$ A. Keren, O. Shafir, E. Shimshoni, V. Marvaud, A. Bachschmidt, and J. Long, Phys. Rev. Lett. 98, 257204 (2007).

${ }^{16}$ F. K. Larsen et al., Angew. Chem. Int. Ed. 42, 101 (2003).

${ }^{17}$ J. van Slageren et al., Chem.-Eur. J. 8, 277 (2002).

${ }^{18}$ S. Piligkos et al., Chem.-Eur. J. 15, 3152 (2009).

${ }^{19}$ The full chemical formulae of the materials studied are $\mathrm{Cr}_{7} \mathrm{Mn}-h=\left[\left(\mathrm{CH}_{3}\right)_{2} \mathrm{NH}_{2}\right]\left[\mathrm{Cr}_{7} \mathrm{MnF}_{8}\left\{\mathrm{O}_{2} \mathrm{CC}\left(\mathrm{CH}_{3}\right)_{3}\right\}_{16}\right] ; \quad \mathrm{Cr}_{7} \mathrm{Mn}-d$ $=\left[\left(\mathrm{C}_{2} \mathrm{D}_{5}\right)_{2} \mathrm{NH}_{2}\right]\left[\mathrm{Cr}_{7} \mathrm{MnF}_{8}\left\{\mathrm{O}_{2} \mathrm{CC}\left(\mathrm{CD}_{3}\right)_{3}\right\}_{16}\right] ; \quad \mathrm{Cr}_{8}-h=\left[\mathrm{Cr}_{8} \mathrm{~F}_{8}\left\{\mathrm{O}_{2}\right.\right.$ $\left.\left.\mathrm{CC}\left(\mathrm{CH}_{3}\right)_{3}\right\}_{16}\right]$; and $\mathrm{Cr}_{8}-d=\left[\mathrm{Cr}_{8} \mathrm{~F}_{8}\left\{\mathrm{O}_{2} \mathrm{CC}\left(\mathrm{CD}_{3}\right)_{3}\right\}_{16}\right]$.

${ }^{20}$ S. J. Blundell, Contemp. Phys. 40, 175 (1999).

${ }^{21}$ R. S. Hayano, Y. J. Uemura, J. Imazato, N. Nishida, T. Yamazaki, and R. Kubo, Phys. Rev. B 20, 850 (1979).

${ }^{22}$ C. P. Slichter, Principles of Magnetic Resonance (SpringerVerlag, New York, 1989).

${ }^{23}$ P. C. E. Stamp and I. S. Tupitsyn, Phys. Rev. B 69, 014401 (2004).

${ }^{24}$ T. Lancaster, S. J. Blundell, P. J. Baker, H. J. Lewtas, W. Hayes, F. L. Pratt, H. T. Yi, and S. W. Cheong, Phys. Rev. B 80, 020409(R) (2009).

${ }^{25}$ P. Santini, S. Carretta, E. Liviotti, G. Amoretti, P. Carretta, M. Filibian, A. Lascialfari, and E. Micotti, Phys. Rev. Lett. 94, 077203 (2005).

${ }^{26}$ F. Troiani, V. Bellini, and M. Affronte, Phys. Rev. B 77, 054428 (2008).

${ }^{27}$ S. J. Blundell, Magnetism in Condensed Matter (OUP, Oxford, 2001). 